\title{
Application of soft sensors in the automated process control of different industries
}

\author{
Oleksandr Savytskyi, Maksym Tymoshenko*, Oleksandr Hramm, and Serhii Romanov \\ Kryvyi Rih National University, Department of Automation, Computer Science and Technology, Kryvyi Rih, 50027, Ukraine
}

\begin{abstract}
Sustainable development of industry is closely related with tries to automate industrial processes in all possible ways. Recent advances in automated control systems have led to decreasing the cost of hardware and energy consumption. This article describes examples of soft sensors using in various industries. The main advantages of soft sensors are low cost, flexibility and versatility. In addition, the soft sensors are environmentally friendly as they significantly reduce the amount of equipment and do not require utilization. Despite these benefits, there are some problems with using them. First problem consists in what information and how needs to be measured to use received data in calculation of another virtual data. Second problem is using proper software and the time of mathematical calculations. The goal of the soft sensors is generation of valid virtual data for the controller to increase the accuracy and quality of the automated control. The article is of interest from the point of view of possibilities to applicate modern technology in solving various tasks of automated control.
\end{abstract}

\section{The rationalisation of using soft sensors in the automated control of industrial sites}

Increasingly, industrial enterprises are demanding improved production efficiency, following government laws that apply strict restrictions on product specifications and pollutant emissions, leading to increasingly effective measurement and control policies. In the context of the sustainable development of industrial process control, the importance of monitoring a large set of processes variables with the help of adequate measuring instruments is clear. However, the key obstacle to implementing a large-scale monitoring and control policy is the high cost of online meters [1-3].

Mathematical process models based on experimental data, through systematic identification procedures, can greatly help to reduce the need for measuring instruments and to develop a rigid management policy. Mathematical models designed for the aforementioned purposes are known as virtual analysers or soft sensors.

Soft sensors are a valuable tool in many industries, including oil refineries, chemical plants, cement kilns, power plants, pulp and paper industries, food processing, nuclear power plants, urban and industrial pollution monitoring. They are used to solve a number of different problems, such as backup system measurement, "what to do" analysis, real-time forecasting for installation monitoring, sensor testing, and fault diagnosis strategies.

Industries are faced with the choice of an appropriate production policy, which is the result of trade-offs number among various constraints. Final product prices and quality are, of course, two relevant and competing factors that can determine an industry's market success. Closely related to such aspects are topics such as energy and raw materials consumption, especially due to the ever-increasing prices for energy resources. Moreover, compliance with safety rules (according to several studies, inadequate management of emergencies is the main cause of industrial losses) and environmental pollution problems add to the complexity of the scenario.

Unfortunately, measuring instruments are generally required to operate in adverse or extreme conditions, which on the one hand requires instruments to meet very limited design standards, while a maintenance protocol must be planned on the other. In any case, the occurrence of unexpected faults cannot be completely avoided. However, some measurement tools can introduce significant delays in application, which may reduce control efficiency. Setting up and maintaining a largescale metering network is never cheap, and the budget you need can significantly affect the total running costs of the plant, which are usually biased to reduce the total number of variables and/or frequency of observations, although in many industrial situations infrequent sampling (lack of online sensors) of some variable processes can present potential performance issues. The typical case is that product quality variables are determined by offline analysis of samples in the laboratory, thus creating a gap and significant delays [4-6].

Soft sensors offer a number of attractive features: - they are an inexpensive alternative to expensive hardware devices, allowing for more comprehensive monitoring networks; 
- they can work in parallel with hardware sensors, providing useful information for troubleshooting tasks, thereby allowing for more robust processes to be implemented;

- they can be easily implemented on existing hardware (such as microcontrollers) and restored when system settings change;

- they allow you to evaluate data in real time, overcoming the time delays introduced by slow hardware sensors (such as gas chromatographs), thereby improving the effectiveness of control strategies.

There are three basic approaches to building soft sensors: mechanistic modeling (physical modeling), multivariate statistics, and artificial intelligence modeling using neural networks, fuzzy logic, and hybrid methods or algorithms. This classification approach does not have to be very rigid, and techniques specific to one approach are often refined using techniques typical of others.

Appropriate empirical models or data-driven models that generate reliable estimates of real-time process variables based on their correlation with other relevant system variables can be useful tools in industrial applications due to the complexity of installation dynamics, which necessitates the use of soft sensors methods and tools.

Let's consider some examples of soft sensors application by well-known scientists are engaged in the research and implementation of soft sensor technology in automated control systems.

In the article [7] use of soft sensors for the estimation of pollutants in the output flow of a Sour Water Stripping plant in Italy is described. The soft sensors have been implemented by nonlinear data-driven approaches with use of deep learning. The deep learning approach allowed for exploiting all recorded historical data, solving the typical problem of data scarcity. As a result, soft sensors based on neural networks were developed and implemented in the enterprise.

The paper [8] introduces a soft sensor for the estimation of the deflection of a polymeric mechanical actuator. The actuator is based on ionic polymer-metal composites (IPMCs). Applications of IPMCs have been proposed in fields such as robotics, surgery, and aerospace. In such application fields, both the size of the actuating system and the complexity are of chief importance. A soft sensor can be preferred to hardware measuring the actuator output, for estimating the actuator motion. Also, low-order models are of interest to limit the computational load, which can be a constraint in realtime applications. To this aim, several data-driven nonlinear finite-impulse response models have been investigated.

The paper [9] describes an interesting approach for designing a data-driven soft sensor for a plant, in the presence of an unknown measurement delay. For the realisation are used deep belief networks. The features, obtained after the unsupervised learning phase, are exploited for estimating the measurement delay. The procedure is applied to the design of a soft sensor for a debutanizer, which is a part of a refinery settled in Sicily.
The paper [10] analyzes a number of strategies that are devoted to improving the generalization capabilities of neural-network-based soft sensors when only small data sets are available. Authors searched for a strategy that is able to cope with the problem of scarcity of experimental data, which often arises in industrial applications. The methods considered were compared in an industrial case study regarding the design of a backup soft sensor for a thermal cracking unit, working in a refinery plant.

In the paper [11] the soft sensor design strategy for an industrial process, via neural NMA model, is described. In details, the hydrogen sulphide in the tail stream of a Sulfur Recovery Unit of a refinery located in Sicily, Italy, is estimated by a soft sensor. It is based on the minimization of the Lipschitz numbers by a Genetic Algorithms approach. A comparative analysis with an empirical model, developed on the basis of suggestions given by plant experts, is included to show the validity of the proposed procedure.

As can be seen, soft sensors are used in different types of industry and are built using different mathematical mechanisms. The following chapters of article discuss in more detail examples of the soft sensors use by the authors in energy management, the automation of iron ore enrichment processes, and the management of alternative energy sources.

\section{Application of soft sensors in the automated control of iron ore enrichment processes}

\subsection{The complexity of controlling the process of loading the mill with iron ore}

Iron ore grinding is a preparatory process for magnetic ore enrichment. Magnetic separation is the main process of magnetizing factories. According to the sustainable development principles in mining and processing it is necessary to build and control systems of magnetprocessing factories technological complexes. The pilot or conductive control object is a magnetic separator, and the driven one is a drum mill, the task of which is to open the spores of a useful mineral with an empty rock. The possible degree of ore opening is determined by the nature and size of the grain entrapment. For example, the source iron ore supplied for grinding, classification and magnetic separation is substantially heterogeneous in its chemical composition and texture and structural properties, even within the same deposit. For example, the Ingulets deposit of the Kryvyi Rih iron ore basin is represented by four types of ores: heavily enriched, consisting of carbonate-magnetite and silicate-magnetite quartzites (magnitude of magnetite incorporation 0.008$0.08 \mathrm{~mm}$ ); medium enriched crushed, consisting of magnetite-silicate and carbonate silicate-magnetite quartzites (magnitude of magnetite incorporation of 0.02-0.2 $\mathrm{mm}$ ); medium-enriched light-crushed, consisting of red-gray-magnetite silicate and silicatemagnetite quartzites medium and broad-banded (magnitude of 0.02-0.07 mm inclusions); light enriched, 
consisting of silicate-magnetite and magneto-silicate quartzites (magnitude of magnetite incorporation of $0.02-0.1 \mathrm{~mm}$ ). Variables are also the strength of the ore and its density, as well as the mineral composition: magnetite, martite, hematite, iron hydroxide, siderite, sulfides, quartz, silicates, etc.

The main methods of ore preparation at the magnetic concentrating factories are grinding and classification in order to control the particle size distribution of the ore supplied to the magnetic enrichment. Given the uneven composition and variable properties of the ore, there is a need to control the grinding process in order to achieve the optimum size of ore grinding and to obtain the optimal fractional composition of raw materials fed to the magnetic separation.

From the point of view of grinding, the main adjustable variable of the drum mills is the loading or filling the mills with ore, which determines the size of the grinding product at the output of the units. The grinding size significantly depends on the initial enrichment. For example, in the dependences of iron content in concentrate $\beta$ and the extraction of iron in concentrate $\varepsilon$ are presented, depending on the size of grinding (Figure 1).

$$
\varepsilon, \beta, \%
$$

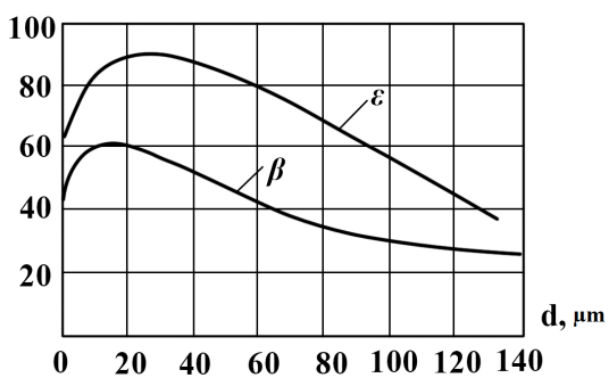

Fig. 1. Dependences of magnetic enrichment indicators according to V. V. Karmazin: $\varepsilon$ - extraction; $\beta$ is the iron content.

The analysis of these dependences shows that there is an optimal size of the mineral opening corresponding to the maximum values of $\varepsilon$ and $\beta$.

The magnetic separator clearly responds to the fractional composition of the mineral raw material (Figure 2). If the raw material is undergrinded, then the iron yield and content in the concentrate will be less than with well-exposed mineral raw materials. Conversely, if the raw material is overgrinded, the content of iron in the tails will increase, the yield of the concentrate will also decrease.

Thus, a magnetic separator is a natural indicator of grinding results. The optimal size of grinding ore will correspond to the optimum magnetic enrichment.

Therefore, the criterion for optimal loading of the drum mill is the maximum performance of the pilot magnetic separator for the magnetic product.

There is a known relationship between the active power consumed by a magnetic separator motor and the separator performance by a magnetic product. So, the criterion for optimal loading of the ore drum mill is the maximum value of the active power of the magnetic separator drum motor.

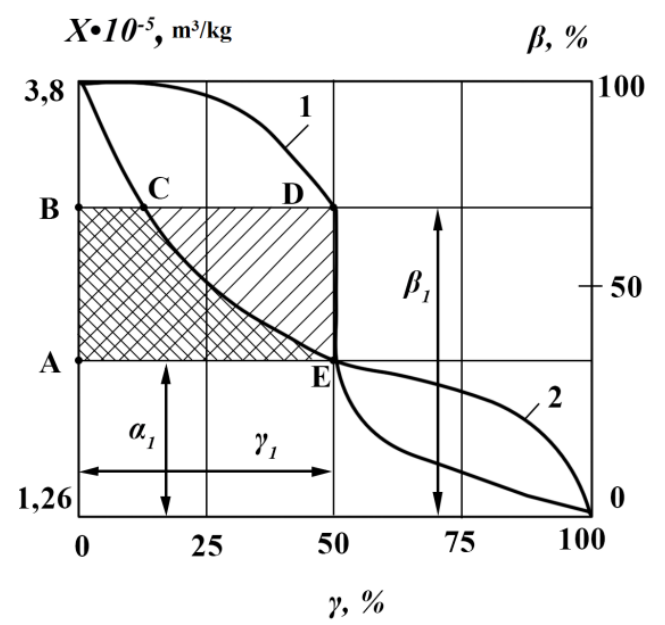

Fig. 2. Enrichment curve for good (1) and bad (2) grinded ore

\subsection{Application of soft sensors for automatic control of ore loading process}

In order to develop algorithms for the optimization of technological complexes of magnetic ore enrichment with ball mills and milling machines, it is necessary to know the static characteristics of these complexes.

A mathematical model of the technological complex of magnetic enrichment of the first stage (Figure 3) was compiled.

Symbols of Figure 3 have description: $\varphi_{p}$ - degree of loading mill with iron ore; $P_{S}-$ active power of the separator motor; $P_{M}-$ active power of the mill motor, $\gamma_{p}$ - pulp density in the mill.

As a result of the simulation, a static characteristic of the technological complex was obtained (Figure 4).

The analysis of this characteristic shows that to the maximum productivity of the complex in the magnetic product, the active capacities consumed by the mill $P_{M}$ and the separator $P_{S}$ are unidirectional, that is, with increasing degree of mill loading with ore they increase. Further increase of loading degree leads to a decrease in the active power consumed by the separators $P_{S}$, and accordingly the productivity of the complex by the magnetic product, while the power consumed by the drive engine of the mill increases.

Similarly, the static characteristics of the technological complex of magnetic enrichment with a ball mill of (Figure 5) were obtained.

During the study, it was assumed that the overflow's density of the classifier was stabilized by an automatic density control system at $1400 \mathrm{~g} / \mathrm{l}$.

On Figure 4 the following designations are accepted: 1 - large interspersed ore; 2 - medium interspersed ore; 3 - little interspersed ore.

On Figure 5 the following designations are accepted: $1-40 \mathrm{t}$ ball load; $2-45 \mathrm{t}$ ball load; $3-50$ ton ball load.

The degree of the ore mill loading $\varphi$ was measured and stabilized at a predetermined level using a radioisotope fill indicator and a mill loading control system. During each experiment, to collect the 
experimental data, the laboratory of the Pivnichnyi GZK (Kryvyi Rih Northern Mining and Processing Plant) selected technological samples of industrial products and tails, performed their chemical analysis of the iron content and determined the volumetric productivity of the separator on the products by measuring capacity and stopwatch, as well as the productivity of the complex by magnitude $Q_{M}$. At the same time, readings of measuring instruments of the drive motor active power of the mill $P_{M}$ and the drive motor active power of the group of magnetic separators of the first stage $P_{S}$ were recorded. The regression equation was obtained

$$
P_{S}=16.7+3.27 Q_{M}
$$

which confirms the possibility of measuring the performance of the technological complex by magnetic iron $Q_{M}$ by the amount of active power consumed by a group of magnetic separators. According to the results of the experiment, the energy consumption per ton of the obtained magnetic product was simulated. The correlation coefficient between the variables $Q_{M}$ and $P_{S}$ was 0.89 .

Since the active power consumed by magnetic separators, $\mathrm{P}_{\mathrm{S}}$ is proportional to the performance of the complex over magnetic iron $Q_{M}$, then the maxima of static characteristics $Q_{M}=f(\varphi)$ and $P_{S}=f(\varphi)$ must coincide. Therefore, an increase in the degree of loading of the ore mill $\varphi$ leads to an increase in the active power $P_{S}$ consumed by the magnetic separators and the active power $P_{M}$ consumed by the mill. Further increase in the degree of mill loading with ore leads to a decrease in the performance of the complex on the magnetic product $Q_{M}$ and, accordingly, consumed active power $P_{S}$ by the separators, while the power $P_{M}$ consumed by the drive engine of the mill, increases.

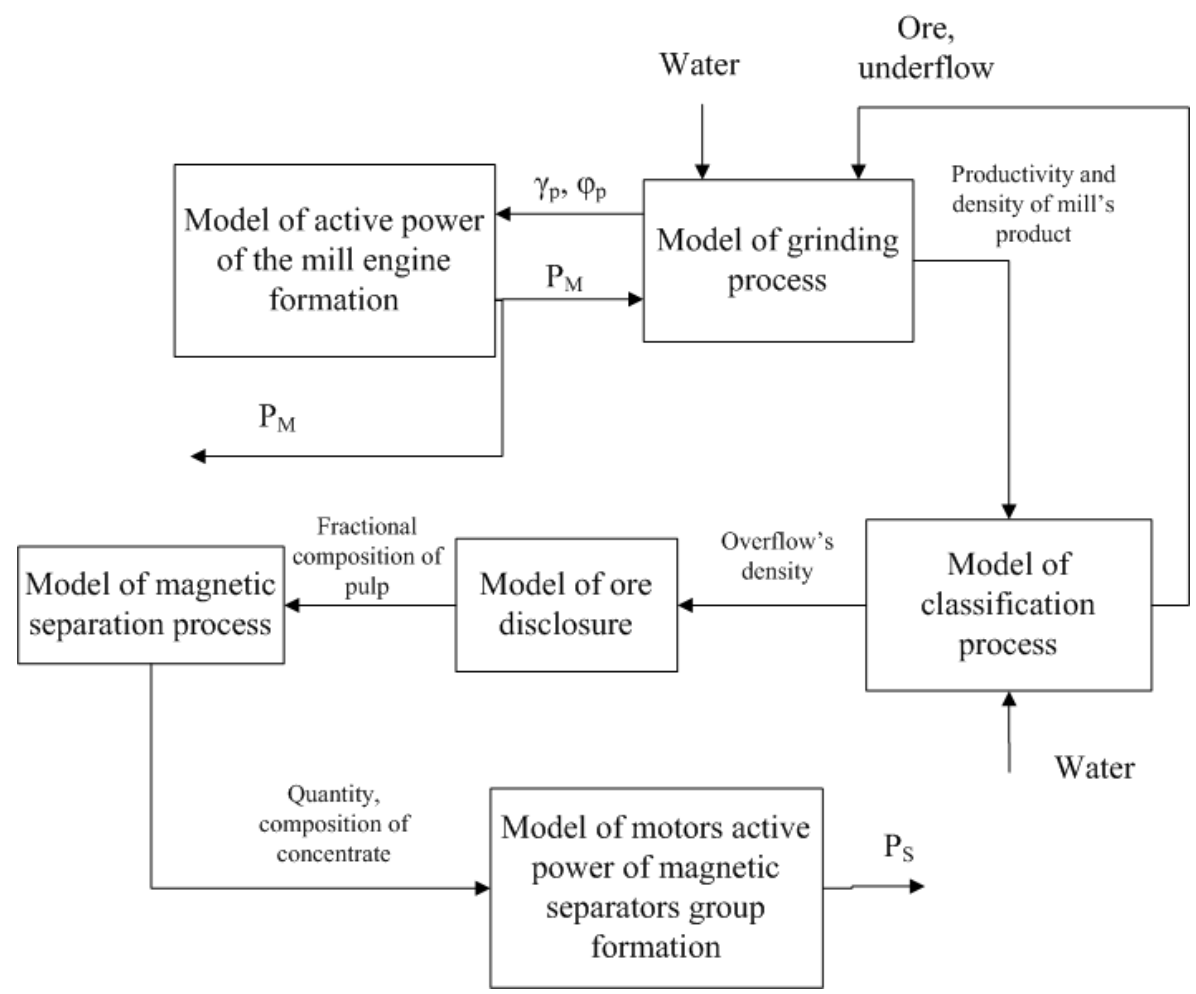

Fig. 3. Scheme of the model of information transfer between mechanisms on the course of the technological complex of magnetic enrichment.

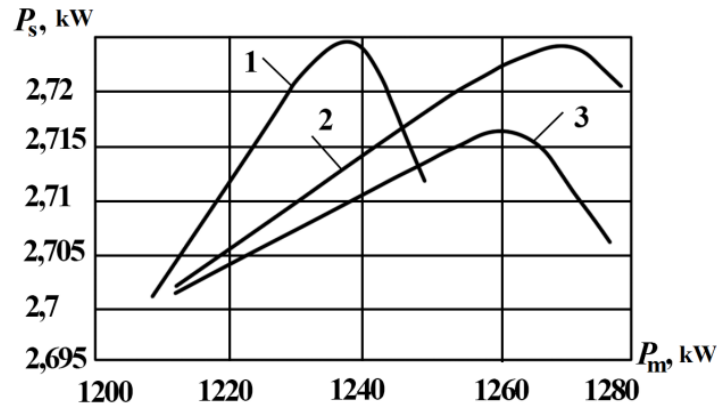

Fig. 4. Static characteristics of a technological complex of magnetic enrichment with a mill of wet selfgrinding.

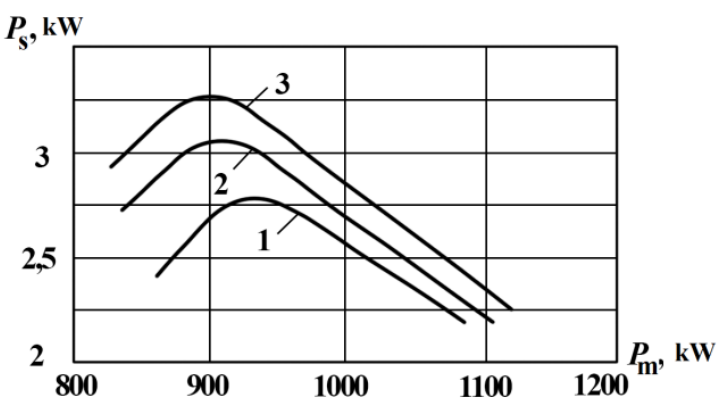

Fig. 5. Static characteristics of the technological complex of magnetic enrichment with a ball mill. 
The conducted researches confirm that it is advisable to use soft sensors to control the process of loading the mill with iron ore, namely to control the degree of the mill loading with ore, given the active power of the magnetic separator motor.

\section{Using of soft sensors in the automated control of solar power plants}

In any real system, such as a solar power plant, a data collection system must be put in place to monitor performance, which provides monitoring of the parameters that are important to the system. There are many methods that use classic hardware to collect and transmit data, but in large solar power plant, this can be quite expensive due to the large amount of sensors required and therefore impractical to implement. In solar plants, such controlled parameters are the output power, voltage and current at the panel output, its temperature, and in the case of solar tracking systems - the angle of inclination to the horizon and the angle of rotation of the panel. Such a system has a complex relationship between all parameters, and therefore the technique of obtaining one or more variables from another measured value can be applied. That is why the use of virtual sensors, or so called soft sensors, is most appropriate in terms of reducing the amount of equipment needed and, as a consequence, its cost.
However, this is not the only one property of soft sensors. If there is already a sufficient set of equipment and sensors in the solar power plant, the use of virtual sensors can improve control accuracy. Sometimes, the sampling rate of real sensors is not high enough, which does not match the capabilities of the controllers. Therefore, by increasing the amount of data received by the controller, it can be improved: the controller receives a signal from real sensors every $T$ seconds, and additionally, from virtual sensors every $T / 5$ seconds. This method, in which the data processing speed of the controller is $N$ times greater than the actual sensor sampling period, is called multi-rate. And at times when real-world sensors can't get information due to certain unforeseen circumstances, soft-sensor information allows the controller to continue its control task at the normal level, the effects of missing information are minimized.

The block diagram of the system using the soft sensor based on a mathematical model of a multi-rate process is shown in Figure 6. In this system, the soft sensor works in parallel with the real sensor, and in realtime receives data from it and additionally receives a control signal coming from the controller to the actuator. The advantage is that the effects of delay in the transmission of the control signal and the sampling rate of the real sensor on the real process are taken into account in the virtual environment, providing more reliable virtual data from the soft sensor.

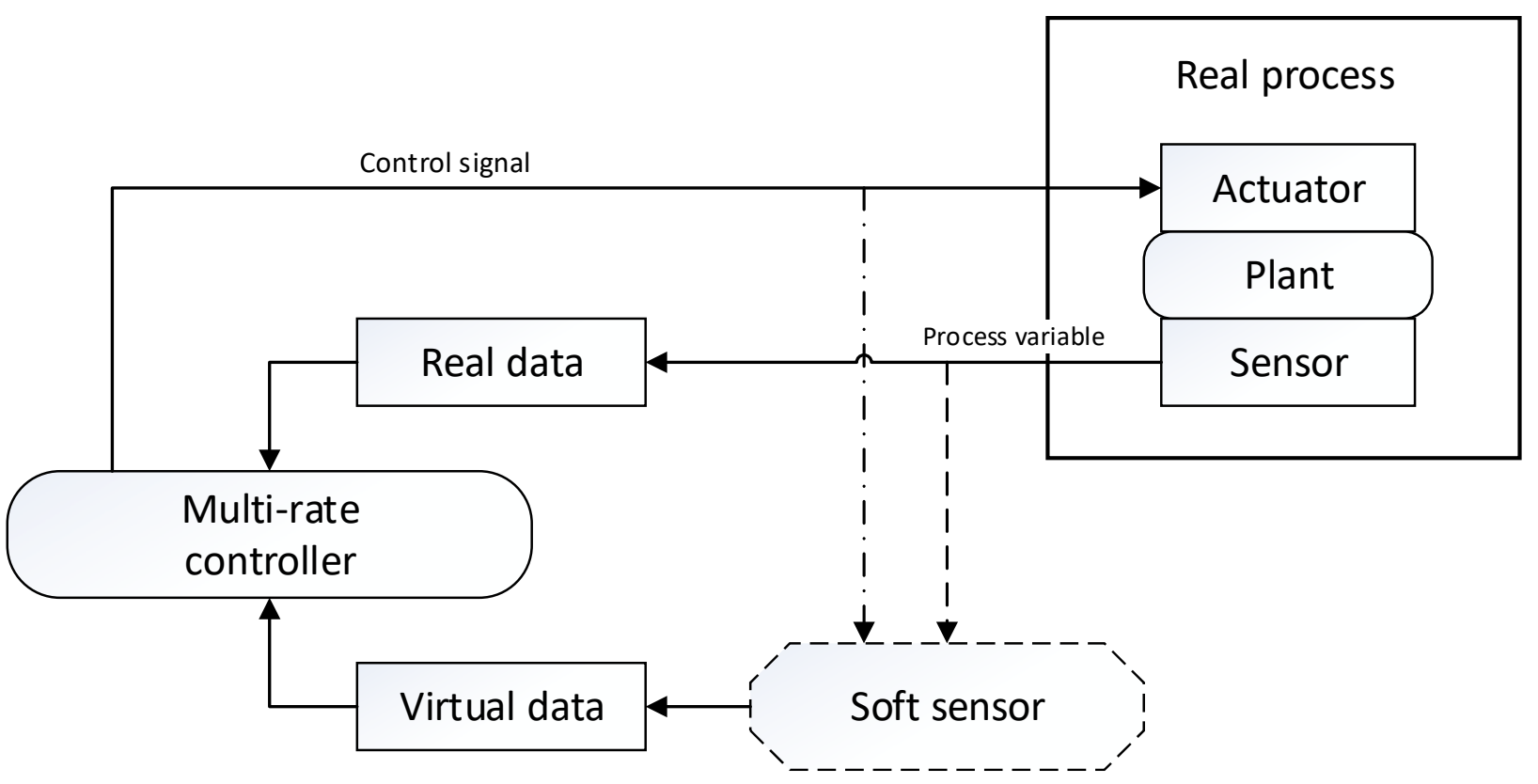

Fig. 6. Block diagram of a soft sensor control system.

According to Figure 6, the multi-rate controller uses virtual data obtained from a soft sensor based on the process model to calculate additional control actions sent to the actuator. Thus, the triggering process is faster than the actual sampling rate of real sensors, overcoming the problem of limiting the sampling rate of those sensors.

As noted above, we are considering the implementation of soft sensors for a solar power plant. One of the most important parameters of this system is the maximum power of the generated electricity. Maximum power point tracking is a must to maximize the efficiency of converting solar energy to electricity using solar panels. Directly the power is affected by the amount of solar irradiance per $1 \mathrm{~m}^{2}$ of solar field: the more solar energy, the greater the output power of the system; and partial shading dramatically reduces the amount of electricity. Therefore, it is important to receive timely data on the level of solar irradiation to 
adjust the position of solar panels. Complex instruments such as pyranometers or pyrheliometers are used to determine the amount of solar radiation per unit area. But their cost is too high to install many sensors across the entire solar power plant. The maximum power of the solar panel directly depends on the performance ratio PR:

$$
P R=\frac{P_{i}}{P_{\text {nom }}} \frac{G_{S T C}}{G_{i}}
$$

where, $P_{i}-$ measured instantaneous power, $P_{\text {nom }}-$ nominal power of the panel, $G_{i}$ - actual instantaneous level of solar radiation, $G_{s t c}-$ solar radiation under normal conditions. Almost all of the values in the (2) are known: the nominal power is given in the solar panel technical data sheet, the instantaneous power can be measured by multiplying the voltage $U_{i}$ and the current $I_{i}$ at the output of the panel, and solar radiation under normal conditions can be obtained from the statistics from the geographical location of the solar power plant. The amount of solar radiation that falls into the panel at the moment can be calculated from formula (2):

$$
G_{i}=\frac{P_{i}}{P_{\text {nom }}} \frac{G_{S T C}}{P R}
$$

where, $P R$ is applied as coefficient of $0.6-0.8$ as for normal panels functioning.

In this way, the soft sensor will calculate the instantaneous solar radiation for the panel positioning system relative to the sun to possibly increase the electricity generated and improve the efficiency of the solar power plant. And in the case of dividing a large field of several hundred or thousands of solar panels into separate sections, calculate the solar radiation for each of them to position these areas independently of each other, to minimize the effects of partial shading of the panels by clouds.

\section{Soft sensors using approach in industrial reactive energy measuring}

Sustainable development of industry is associated with increasing requirements for the quality of the supplied electric energy. Moreover, a very important factor is the ability to accurately measure the power flowing in the network at any level. This is important for both suppliers and consumers of energy. With increasing productivity and improving the quality of finished products of industrial enterprises, their consumption of energy resources also grows. At the same time, the requirements for the capacity of the power lines of these objects are increasing. The observed trend of a significant increase in the share of non-linear and non-stationary loads in power networks leads to a reduction in the throughput of these distribution networks due to an increase in the reactive capacities flowing in them. This problem is associated with the increasing use of power electronics devices among other consumers of electric energy. Examples of such devices include induction furnaces and heaters, gas discharge lamps, variable speed drives, etc.
The growth of reactive power flowing in the enterprise network creates a large amount of interference for the enterprise itself and adversely affects the entire distribution network. Also, the fact that such consumers are becoming generally accepted leads to the fact that the equipment on the consumer side and on the side of the electric energy suppliers operates in non-sinusoidal signal modes, which adversely affects the accuracy of measuring instruments. And in conjunction with the growth of reactive power flowing in the network, it leads to an increase in losses during the transportation of electric energy and a decrease in the power factor of consumers. The measurement and control of the adequacy of the reactive power measurement system is associated with a set of problems, including the high cost of such a system for large industrial facilities, such as mining and processing plants, because of their distributed structure and the supply of different structural units of such an object to different power lines from different distribution substations. In such a situation, as a possible solution to this problem, we can consider the analytical calculation of reactive power volumes through other simply measured parameters of the distribution network. So when considering the distribution system of electric energy and the nature of the loads connected to it as linear and operating exclusively in the mode of sinusoidal signals, the reactive power is determined by a simple ratio:

$$
Q=U \times I \times \sin \varphi
$$

Where: $I$ is the effective value of the current strength in the network; $U$ is the effective voltage value in the network; $\varphi$ is the phase difference between current and voltage.

When considering the operation mode of the system under the influence of non-harmonic signals, the effective values of voltage and current in the network can be expressed as:

$$
\begin{gathered}
I=\sum_{k=1}^{K} I_{k} \sin \left(2 \pi k f_{0} t-\psi_{k}\right) \\
U=\sum_{k=1}^{K} U_{k} \sin \left(2 \pi k f_{0} t-\theta_{k}\right)
\end{gathered}
$$

Where: $k$ is the harmonic number of the sinusoidal signal of frequency $f_{0} ; I_{k}$ is the effective current value of the $k$ harmonic; $U_{k}$ is the effective voltage value of the $k$ harmonic; $\psi_{k}$ and $\theta_{k}$ are the initial phases of the signals; $K$ is the total number of harmonics considered.

As can be seen from the above expressions, the analytical solution to the problem posed is associated with an even greater number of difficulties due to the large number of additional difficult to measure quantities. Based on this, it seems logical to use soft sensors for calculating reactive power. Let us consider as an object the mining and processing plant mentioned above. We make the assumption that, within the framework of one distribution substation, the consumption of reactive power by each structural element of the object affects the consumption of the others. In this case, the structural element with the largest volumes of reactive energy consumption should have the greatest influence. To verify this assumption, 
we use statistics on the consumption of reactive energy of the mining and processing plant. There are statistical data on the consumption of reactive energy by the four structural elements of the facility, which are supplied with electrical energy from one distribution substation. A fragment of statistical data on the consumption of reactive power by four structural elements of an object with a measurement frequency of two hours during one day is given in the Table 1 .

For a complete set of available statistical data, we will calculate the correlation coefficient of the reactive power consumption of the structural element with the highest consumption of other structural elements. The calculation was carried out according to statistical data for 7 calendar days. The result obtained in the form of correlation coefficients of reactive power consumption by objects 2-4 with object 1 for a period of 7 days is displayed in the Table 2 .

Table 1. Statistical data of mining and processing plant reactive power consumption.

\begin{tabular}{|c|c|c|c|c|}
\hline Time, h & $\begin{array}{c}\text { Obj. 1, } \\
\text { kvar*h }\end{array}$ & $\begin{array}{c}\text { Obj. 2, } \\
\text { kvar*h }\end{array}$ & $\begin{array}{c}\text { Obj. 3, } \\
\text { kvar*h }\end{array}$ & $\begin{array}{c}\text { Obj. 4, } \\
\text { kvar*h }\end{array}$ \\
\hline $02: 00$ & 14348 & 46 & 102 & 972 \\
\hline $04: 00$ & 13218 & 45 & 53 & 912 \\
\hline $06: 00$ & 14649 & 47 & 102 & 912 \\
\hline $08: 00$ & 14471 & 49 & 106 & 792 \\
\hline $10: 00$ & 13771 & 50 & 101 & 80 \\
\hline $12: 00$ & 11534 & 48 & 96 & 804 \\
\hline $14: 00$ & 12790 & 43 & 127 & 1020 \\
\hline $16: 00$ & 13077 & 48 & 98 & 1224 \\
\hline $18: 00$ & 12552 & 43 & 98 & 804 \\
\hline $20: 00$ & 12344 & 5 & 119 & 996 \\
\hline $22: 00$ & 13760 & 5 & 94 & 888 \\
\hline $24: 00$ & 12400 & 6 & 95 & 732 \\
\hline
\end{tabular}

Table 2. Correlations coefficients of structural elements reactive power consumption

\begin{tabular}{|c|c|c|c|}
\hline Day & Element 2 & Element 3 & Element 4 \\
\hline 1 & 0,569 & 0,303 & 0,659 \\
\hline 2 & 0,741 & 0,469 & 0,897 \\
\hline 3 & 0,433 & 0,498 & 0,965 \\
\hline 4 & 0,565 & 0,443 & 0,759 \\
\hline 5 & 0,728 & 0,248 & 0,903 \\
\hline 6 & 0,715 & 0,645 & 0,796 \\
\hline 7 & 0,638 & 0,459 & 0,956 \\
\hline
\end{tabular}

From the obtained correlation coefficients, we can conclude that the initial statement about the relationship between reactive power consumption by the structural elements of the mining and processing plant within the boundaries of one distribution substation is correct, and the obtained data can be used to further search for mathematical patterns and develop a soft sensor to predict the reactive power consumption value of all structural objects of the mining and processing plant that are supplied with electrical energy from one distribution substation based on the value of reactive power consumption of one of these elements.

\section{Analyse of obtained results}

The analysis of soft sensors using in the automated control of various industrial processes shows their versatility and benefits. The use of soft sensors is often a more viable alternative to hardware, as very often physical sensors have to operate in extreme conditions, are quite expensive and need frequent replacement. It is advisable to use soft sensors in the case of a large number of similar mechanisms operating in approximately the same conditions. Measuring certain performance of one mechanism, you can roughly judge the situation as a whole.

Unlike sensors made from various material substances, soft sensors have a different basis. By definition, "soft sensors" are based on the use of software and the calculation of regulated process parameters. Thus, the environmental friendliness of their use is not in doubt.

At the same time, it is not necessary to check such sensors for the content of harmful substances and hazardous emissions during their operation. At the end of their life cycle, one does not have to worry about compulsory utilization.

As for the status of a sustainable product, the application of software solutions has a long-term perspective on sustainable development.

Soft sensors can be built on different technologies. These can be physical implementations that combine several heterogeneous primary and secondary converters in their design. But more often, soft sensors are modelled virtually on the basis of mathematical devices of fuzzy logic, neural networks, adaptive systems, etc. The choice of mathematical apparatus depends entirely on the specific situation and the specific task.

The main difficulty in working with the principles of soft sensors is lack of knowledge about them due to their novelty and, as a consequence, the lack of conventional mathematical descriptions or structure. On the other hand, it opens up opportunities for creativity.

In general, to work with soft sensors it is necessary to have large arrays of statistics for calculations. It is necessary to have a good understanding of the controlled process principles, physical features and find correlation between the parameters.

The review results suggest that the soft sensor instruments can significantly reduce the amount of mechanical equipment and, through calculated correlation dependencies, delegate the task of measuring certain process parameters to software.

\section{References}

1. B. Andò, S. Graziani, M.G. Xibilia, A soft sensor for the estimation of ionic electroactive actuator motion based on deep learning, in 2018 IEEE International Instrumentation and Measurement Technology Conference (I2MTC), pp. 1-6

2. M.G. Xibilia, N. Gemelli, G. Consolo, Input variables selection criteria for data-driven Soft Sensors design, in 2017 IEEE 14th International 
Conference on Networking, Sensing and Control (ICNSC), pp. 362-367

3. G.Napoli, M.G. Xibilia, Soft Sensor design for a Topping process in the case of small datasets. Computers \& chemical engineering 35(11), 2447$2456(2011)$

4. R. Caponetto, G. Dongola, A. Gallo, M. G. Xibilia, FPGA Implementation of a soft sensor for the estimation of the freezing point of kerosene, in ASME 2009 International Design Engineering Technical Conferences and Computers and Information in Engineering Conference, pp. 151158

5. L. Fortuna, S. Graziani, M.G. Xibilia, Soft sensors for product quality monitoring in debutanizer distillation columns. Control Engineering Practice 13, 499-508 (2005)

6. L. Fortuna, S. Graziani, M.G. Xibilia, Virtual instruments in refineries. IEEE Instrumentation \& Measurement Magazine 8, 26-34 (2005)

7. S. Graziani, M.G. Xibilia, A deep learning based soft sensor for a sour water stripping plant, in 2017 IEEE International Instrumentation and Measurement Technology Conference (I2MTC), pp. 1-6

8. B. Andò, S. Graziani, M.G. Xibilia, Low-order nonlinear finite-impulse response soft sensors for ionic electroactive actuators based on deep learning. IEEE Transactions on Instrumentation and Measurement 68(5), 1637-1646 (2018)

9. S. Graziani, M.G. Xibilia, Design of a soft sensor for an industrial plant with unknown delay by using deep learning, in 2019 IEEE International Instrumentation and Measurement Technology Conference (I2MTC), pp. 1-6

10. L. Fortuna, S. Graziani, M.G. Xibilia, Comparison of soft-sensor design methods for industrial plants using small data sets. IEEE Transactions on Instrumentation and Measurement 58(8), 2444-2451 (2009)

11. A. Di Bella, L. Fortuna, S. Graziani, G. Napoli, M. G. Xibilia, Soft sensor design for a sulfur recovery unit using genetic algorithms, in 2007 IEEE International Symposium on Intelligent Signal Processing, pp. 1-6 